\title{
Caracterização fenotípica e molecular de acessos de mandioca de indústria com potencial de adaptação às condições do Cerrado do Brasil Central
}

\section{Phenotypic and molecular characterization of industrial purpose cassava accessions with potential for adaptation to the conditions of Cerrado of Central Brazil}

\author{
Eduardo Alano Vieira ${ }^{1 *}$; Josefino de Freitas Fialho'; Fabio Gelape Faleiro ${ }^{1}$; \\ Graciele Bellon ${ }^{2}$; Kenia Graciele da Fonseca ${ }^{2}$; Marilia Santos Silva ${ }^{1}$; \\ Silvana Vieira de Paula-Moraes ${ }^{1}$; Luiz Joaquim Castelo Branco Carvalho ${ }^{3}$
}

\section{Resumo}

\begin{abstract}
O objetivo do trabalho foi caracterizar e estimar a variabilidade genética entre acessos de mandioca de indústria, com potencial de adaptação às condições do Cerrado do Brasil Central, por meio de caracteres quantitativos, qualitativos e marcadores moleculares de forma isolada e conjunta, bem como estimar a correlação entre as estimativas obtidas com base em cada grupo de caracteres. Dezesseis acessos de mandioca de indústria com potencial de adaptação às condições do Cerrado do Brasil Central foram avaliados a campo quanto a 11 caracteres quantitativos e 33 caracteres qualitativos, em experimento conduzido na Embrapa Cerrados. Os acessos foram também avaliados quanto a marcadores RAPD em laboratório. Posteriormente, foram estimadas as matrizes de dissimilaridade/distância genética entre os acessos por meio dos caracteres qualitativos, quantitativos e marcadores moleculares e pela análise conjunta dos dados. Além disso, foi estimada a correlação entre as matrizes. No grupo de acessos de indústria avaliados existe ampla variabilidade quanto aos caracteres quantitativos, qualitativos e moleculares aferidos. As únicas correlações significativas encontradas foram entre (i) a matriz de dissimilaridade estimada por meio da análise conjunta e a matriz de dissimilaridade estimada por meio de dados qualitativos $(\mathrm{r}=0,52)$ e; (ii) a matriz de dissimilaridade estimada por meio da análise conjunta e a matriz de dissimilaridade estimada por meio de marcadores moleculares $(\mathrm{r}=0,75)$. A fraca associação entre essas medidas indicou que a melhor estratégia para orientar ações de conservação e uso de germoplasma de mandioca de indústria é por meio de estudos de divergência genética com o emprego de marcadores moleculares, caracteres qualitativos e caracteres quantitativos de forma conjunta e complementar.
\end{abstract}

Palavras-chave: Manihot esculenta Crantz, recursos genéticos, melhoramento genético, distância genética, amido

\footnotetext{
1 Eng ${ }^{\text {os }}$ Agr $^{\text {os }}$, Pesquisadores da Embrapa Cerrados, Planaltina, DF. E-mail: eduardo.alano@embrapa.br; josefino.fialho@embrapa. br; marilia.silva@embrapa.br; silvana.moraes@embrapa.br; fabio.faleiro@embrapa.br

2 Eng ${ }^{\text {os }}$ Agr $^{\text {os }}$, Bolsista da Embrapa Cerrados, Planaltina, DF. E-mail: graciele.bellon@colaborador.embrapa.br; keniagro@yahoo. com.br

3 Eng ${ }^{\circ}$ Agr $^{\circ}$, Pesquisador da Embrapa Cenargen, Brasília, DF. E-mail: luiz.castelo@embrapa.br

* Autor para correspondência
} 


\begin{abstract}
The aim of the present work was to characterize and estimate the genetic variability among industrial purpose cassava accessions, with potential for adaptation to the conditions of Cerrado of Central Brazil, by means of quantitative and qualitative characters, and molecular markers through isolated and joint analysis, as well as to establish the correlation among the estimated indexes obtained by each used methodology. Sixteen industrial purpose cassava accessions with potential for adaptation to the conditions of Cerrado of Central Brazil were evaluated in field conditions in terms of 11 quantitative characters and 33 qualitative characters, in an experiment carried out at Embrapa Cerrados. The accessions were also evaluated through RAPD markers in laboratorial conditions. Afterwards, the matrices of genetic dissimilarity/distance among the accessions were estimated through qualitative characters, quantitative characters and molecular markers, besides through the joint analysis of the obtained data. Moreover, the association among the matrices was estimated. The results revealed existence of high variability among the accessions in terms of quantitative, qualitative and molecular characters evaluated. The only significant correlations found were between (i) the dissimilarity matrix estimated through joint analysis and the dissimilarity matrix estimated through qualitative data $(r=0,52)$; and (ii) the dissimilarity matrix estimated through joint analysis and the dissimilarity matrix estimated through molecular markers $(\mathrm{r}=$ $0,75)$. The weak association between these measures indicated that the best strategy to guide conservation actions and use of cassava industry germplasm is through studies of genetic divergence by the use of molecular markers, qualitative and quantitative traits in a joint and complementary way.
\end{abstract}

Key words: Manihot esculenta Crantz, genetic resources, breeding, genetic distance, starch

\section{Introdução}

No Brasil, provável centro de origem e de diversidade da mandioca (Manihot esculenta Crantz) (OLSEN, 2004), a espécie é cultivada de Norte a Sul, revelando grande plasticidade genética, capacidade de adaptação a diferentes condições climáticas e rusticidade. A mandioca é cultivada, principalmente, em razão de suas raízes ricas em amido, que podem ser consumidas na alimentação humana e animal além de apresentarem amplo emprego industrial. O produto industrializado da mandioca com maior potencial de comercialização em larga escala e de exportação é a fécula, cuja produção no Brasil está concentrada nos Estados do Paraná, São Paulo e Mato Grosso (VILPOUX, 2008).

Nos últimos anos, a região do Cerrado, maior região produtora de grãos do Brasil, vêm atraindo a instalação de grandes fecularias e, como conseqüência, o cultivo de mandioca de indústria vem se profissionalizando na região. Essa perspectiva de mercado tem levado a uma forte demanda dos produtores por variedades de mandioca de indústria específicas para a região e com maior qualidade e potencial produtivo.
A caracterização do germoplasma de mandioca de indústria disponível é fundamental para sua utilização no melhoramento genético e para definição de estratégias para sua conservação. Nesse sentido, a estimativa das distâncias genéticas entre um conjunto de acessos é uma ferramenta auxiliar de grande importância em programas de melhoramento e um importante elo entre a conservação e a utilização dos recursos genéticos disponíveis (MOHAMMADI; PRASANNA, 2003). A análise da variabilidade genética fornece informações úteis sobre a organização do germoplasma, aumenta a eficiência da amostragem de genótipos, auxilia na definição de genitores em cruzamentos artificiais, na incorporação de genes de germoplasma exótico e até na recomendação de cultivares para determinada região, quando o objetivo é aumentar a base genética das cultivares a serem cultivadas.

Dentre as ferramentas utilizadas para estudar a variabilidade genética, destacam-se a utilização isolada de marcadores moleculares (CARVALHO; SCHAAL, 2001; FERREIRA et al., 2008) ou caracteres fenotípicos qualitativos e quantitativos (MKUMBIRA et al., 2003; NICK et al., 2008). Dentre os caracteres fenotípicos, os quantitativos 
apresentam a maior influência do ambiente por serem governados por vários genes. Entretanto, esses caracteres são fundamentais na caracterização de acessos, uma vez que vão refletir o real potencial produtivo dos acessos e a possibilidade de utilização de forma direta ou no melhoramento genético. Por sua vez, os caracteres qualitativos têm a sua importância na caracterização de germoplasma por serem relativamente de fácil aferição, de menor custo e menos influenciados pelo ambiente.

Os marcadores moleculares, em relação aos caracteres fenotípicos, apresentam a vantagem de não sofrerem influência do ambiente, porém, apresentam a desvantagem de acessarem o genoma como um todo e não somente as regiões responsáveis pela manifestação dos caracteres de interesse. Dentre os marcadores moleculares, o RAPD (polimorfismo de DNA amplificado ao acaso) merece destaque, pelo baixo custo, pela rápida e fácil execução e pelo fato de não exigir a síntese de iniciadores específicos. Dessa forma vem sendo amplamente utilizado na caracterização de germoplasma de mandioca (CARVALHO; SCHAAL, 2001; ASANTE; OFFEI, 2003; ZACARIAS et al., 2004; VIEIRA et al., 2008a).

Apesar da importância da análise da variabilidade genética, não existem relatos na literatura destes estudos entre acessos de mandioca de indústria, por meio da utilização de marcadores moleculares, caracteres qualitativos, caracteres quantitativos de forma conjunta e complementar, a fim de orientar estratégias de conservação e de uso de germoplasma.

Neste trabalho, objetivou-se caracterizar e estimar a variabilidade genética entre acessos de mandioca de indústria, com potencial de adaptação às condições do Cerrado do Brasil Central, por meio de caracteres quantitativos, qualitativos e marcadores moleculares de forma isolada e conjunta, bem como estimar a correlação entre as estimativas obtidas com base em cada grupo de caracteres.

\section{Material e Métodos}

Foram analisados dezesseis acessos de mandioca de indústria mantidos no Banco Regional de Germoplasma de Mandioca do Cerrado (BGMC), sendo quatro desenvolvidos pelo programa de melhoramento genético de mandioca do Instituto Agronômico de Campinas (IAC), oito desenvolvidos pelo programa de melhoramento genético de mandioca da Embrapa Mandioca e Fruticultura Tropical (CNPMF) e quatro variedades locais, selecionadas pela Embrapa Cerrados em razão de apresentarem adaptação às condições do Cerrado Brasileiro, como variedades para indústria (Tabela 1) (VIEIRA et al., 2008b).

Entre 27 de setembro de 2006 e 26 de março de 2008, foi instalado o experimento de campo, em área experimental da Embrapa Cerrados no município de Planaltina, Distrito Federal, Brasil (15 35'30" de latitude Sul, $47^{\circ} 42$ ' 30' de longitude oeste a $1000 \mathrm{~m}$ de altitude). O delineamento experimental foi o de blocos casualizados com três repetições, sendo cada parcela constituída por 4 linhas de 10 plantas, em espaçamento de 1,20 $\mathrm{m}$ entre linhas e $0,80 \mathrm{~m}$ entre plantas, e a área útil constituída pelas 16 plantas centrais de cada parcela. A seleção do material de propagação e os tratos culturais seguiram as recomendações do sistema de produção de mandioca para a região do Cerrado (SOUZA; FIALHO, 2003), tendo sido efetuada a poda da parte aérea a $10 \mathrm{~cm}$ do solo, em todos os acessos, aos 12 meses após o plantio. 
Tabela 1. Acessos de mandioca de indústria analisados com respectivos nomes comuns e locais de coleta/procedência (LCP).

\begin{tabular}{|c|c|c|}
\hline Acessos & Nome comum & LCP \\
\hline BGMC 788 & IAC 14 & IAC - São Paulo* \\
\hline BGMC 1286 & Roxinha & Santa Catarina \\
\hline BGMC 991 & Clone 03 & CNPMF - Bahia** \\
\hline BGMC 1270 & IAC 15 & IAC - São Paulo* \\
\hline BGMC 1297 & BRS Mani-Branca & CNPMF - Bahia** \\
\hline BGMC 996 & Clone 09 & CNPMF - Bahia** \\
\hline BGMC 1304 & Clone 9661/06 & CNPMF - Bahia ${ }^{* *}$ \\
\hline BGMC 1265 & Fibra & Paraná \\
\hline BGMC 1262 & Enita Brava & Tocantins \\
\hline BGMC 923 & BRS Formosa & CNPMF - Bahia** \\
\hline BGMC 1298 & Clone 9794/06 & CNPMF - Bahia* \\
\hline BGMC 1130 & IAC 13 & IAC - São Paulo* \\
\hline BGMC 1299 & CNPMF 9607/7 & CNPMF - Bahia** \\
\hline BGMC 436 & IAC 12 & IAC - São Paulo* \\
\hline BGMC 1107 & Fécula Branca & Paraná \\
\hline BGMC 1305 & Clone 9688/07 & CNPMF - Bahia** \\
\hline
\end{tabular}

*= Programa de melhoramento genético de mandioca do Instituto Agronômico de Campinas (IAC), baseado em Campinas (SP); ${ }^{* *}$ Programa de melhoramento genético de mandioca da Embrapa Mandioca e Fruticultura Tropical, baseado em Cruz das Almas (BA). Fonte: Elaboração dos autores.

Os caracteres quantitativos aferidos foram: i) altura da planta em m (AP); ii) massa da parte aérea sem a cepa em $\mathrm{kg} \mathrm{ha}^{-1}$ (MPA), iii) massa da cepa em $\mathrm{kg} \mathrm{ha}^{-1}(\mathrm{MC})$, iv) produtividade de raízes em $\mathrm{kg} \mathrm{ha-1}$ (PR); v) porcentagem de amido nas raízes por meio do método da balança hidrostática (AM); vi) comprimento do lóbulo da folha em $\mathrm{cm}$ (CLF) obtido por meio da aferição de uma folha do terço médio de cada planta da área útil da parcela seis meses após o plantio; vii) largura do lóbulo da folha em cm (LLF) obtida por meio da aferição de uma folha do terço médio de cada planta da área útil da parcela seis meses após o plantio; viii) comprimento do pecíolo em cm (CP) obtido por meio da aferição de uma folha do terço médio de cada planta da área útil da parcela seis meses após o plantio; ix) número médio de ninfas e adultos de percevejo-de-renda (NMP) obtido por meio da contagem do número de ninfas e de adultos de percevejos-de-renda em três avaliações (26/12/06, 08/01/07 e 26/02/07) em três plantas amostradas por parcela; $\mathrm{x}$ ) incidência de bacteriose (Xantomonas axonopodis pv manihotis) em porcentagem (IB) obtido por meio da contagem do número total de plantas com sintomas da moléstia nas parcelas em duas avaliações (13/03/07 e 18/05/07); e xi) severidade da bacteriose (SB) por meio da avaliação de três plantas por parcela, em duas avaliações (13/03/07 e 18/05/07), utilizandose a escala de notas descrita por Ramos e Takatsu (1987), que varia de 1 a 5, onde: 1 = não há sintomas visíveis; 2 = há apenas manchas foliares; 3 = há manchas foliares e pus no caule; $4=$ há manchas foliares, pus no caule e desfolha; $5=$ há morte descendente. Os dados obtidos foram submetidos à análise de variância e as médias foram agrupadas por meio do teste de comparação de médias de Scott e Knott (1974) a 5\% de probabilidade de erro com o auxílio do programa computacional Genes (CRUZ, 2001). Os dados de SB, para atender os requisitos de homogeneidade de variância e uniformidade de erros, foram transformados para raiz quadrada de $\mathrm{x}$ antes da análise de variância. Posteriormente foi estimada a distância generalizada de Mahalanobis $\left(D^{2}\right)$, entre os 16 acessos de mandioca, com base nos 11 caracteres quantitativos aferidos, com o auxílio do programa computacional Genes (CRUZ, 2001). 
Os acessos também foram caracterizados por meio de 33 caracteres qualitativos aferidos conforme recomendações de Fukuda e Guevara (1998), sendo eles: i) cor da folha apical; ii) pubescência do broto apical; iii) forma do lóbulo central; iv) cor do pecíolo; v) cor do córtex do caule; vi) cor externa do caule; vii) comprimento da filotaxia; viii) presença de pedúnculo nas raízes; ix) cor externa da raiz; x) cor do córtex da raiz; xi) cor da polpa da raiz; xii) textura da epiderme da raiz; xiii) presença de floração; xiv) cor da folha desenvolvida; xv) número de lóbulos; xvi) cor da epiderme do caule; xvii) hábito de crescimento do caule; xviii) cor dos ramos terminais nas plantas adultas; xix) constrições da raiz; xx) cor da nervura; xxi) posição do pecíolo; xxii) proeminência das cicatrizes foliares; xxiii) comprimento das estípulas; xxiv) margem das estípulas; xxv) hábito de ramificação; xxvi) sinuosidade do lóbulo foliar; xxvii) forma da raiz; xxviii) tipo de planta; xxix) comprimento médio da raiz; xxx) diâmetro médio da raiz; xxxi) destaque da película da raiz; xxxii) destaque do córtex da raiz; e xxxiii) posição das raízes. Com base nos caracteres qualitativos que evidenciaram variabilidade entre os acessos, foi estimada a dissimilaridade genética entre os acessos, por meio do complemento do índice de coincidência simples, com auxílio do programa Genes (CRUZ, 2001).

Amostras de DNA genômico utilizadas para obtenção de marcadores RAPD, foram extraídas de folhas em estágio intermediário de maturação, por meio do método do CTAB, com modificações propostas por Faleiro et al. (2003) e quantificado com base na absorbância a $260 \mathrm{~nm}$ e a análise de pureza com base na relação de absorbância a 260 e 280nm. As amostras de DNA de cada acesso foram amplificadas para obtenção de marcadores RAPD conforme descrito por Costa et al. (2005). $\mathrm{Na}$ obtenção dos marcadores foram utilizados 11 iniciadores decâmeros da Operon Technologies Inc., Alameda, CA, EUA: OPD 02, OPE 12, OPF 08, OPG $(05,08,09$ e 15) e OPH $(04,13$ e 15). Os fragmentos amplificados foram separados eletroforeticamente por quatro horas, a 90 volts, em gel de agarose $(1,2 \%)$, corado com brometo de etídio, submerso em tampão TBE (Tris-Borato $90 \mathrm{mM}$, EDTA $1 \mathrm{mM}$ ). Ao término da corrida, os géis foram fotografados sob luz ultravioleta e os marcadores RAPD foram classificados pela presença (1) ou ausência (0) de bandas. As matrizes de dados obtidas foram utilizadas na estimativa da dissimilaridade genética entre os acessos, com base no complemento do coeficiente de similaridade de Jaccard (JACCARD, 1908) e na estimativa do número mínimo de marcadores informativos com o auxílio do programa Genes (CRUZ, 2001).

Com base na análise conjunta dos dados obtidos, foi estimada a dissimilaridade genética entre todos os pares de genótipos por meio do complemento do índice de similaridade proposto por Gower (1971), com o auxílio do programa Multiv v. 2.3 (PILLAR, 1997), índice que se caracteriza por utilizar de forma conjunta dados binários de marcadores moleculares, dados qualitativos e quantitativos na estimativa de um índice único de similaridade que varia de 0 a 1 .

Com base nas matrizes de distâncias/ dissimilaridades genéticas obtidas a partir da análise com caracteres quantitativos $\left(\mathrm{MD}_{\mathrm{QT}}\right)$, qualitativos $\left(\mathrm{MD}_{\mathrm{QL}}\right)$, marcadores RAPD ( $\left.\mathrm{MD}_{\mathrm{RAPD}}\right)$ e da análise conjunta de caracteres qualitativos, quantitativos e marcadores RAPD $\left(\mathrm{MD}_{\mathrm{QT}+\mathrm{QL}+\mathrm{RAPD}}\right)$, foram confeccionados quatro dendrogramas, utilizandose como critério de agrupamento o método baseado na distância média (UPGMA). O ajuste entre as matrizes de distância/dissimilaridade e os respectivos dendrogramas foram estimados pelo coeficiente de correlação cofenética (r), conforme Sokal e Rohlf (1962), com o auxílio do programa computacional NTSYS pc 2.1 (ROHLF, 2000).

A estimativa da significância da associação entre as matrizes de distâncias obtidas por meio da análise de caracteres quantitativos, caracteres qualitativos, marcadores RAPD e da análise conjunta, foi realizada por meio do teste de comparação de matrizes de Mantel, com 1000 permutações (MANTEL, 1967), com o auxílio do programa NTSYS pc 2.1 (ROHLF, 2000). 
Tabela 2. Comparação de médias e resumo da análise de variância dos caracteres altura da planta em m (AP), massa da parte aérea sem a cepa em $\mathrm{kg} \mathrm{ha}^{-1}$ (MPA), massa da cepa em kg ha-1 (MC), produtividade de raízes em $\mathrm{kg} \mathrm{ha}^{-1}(\mathrm{PR})$, porcentagem de amido nas raízes (AM), número médio de ninfas e adultos de percevejo-de-renda (NMP), incidência de bacteriose em porcentagem (IB), severidade de bacteriose (SB), comprimento do lóbulo da folha em cm (CLF), largura do lóbulo da folha em $\mathrm{cm}$ (LLF) e comprimento do pecíolo em $\mathrm{cm}(\mathrm{CP})$ avaliados em dezesseis acessos de mandioca de indústria.

\begin{tabular}{|c|c|c|c|c|c|c|c|c|c|c|c|}
\hline Acesso & $\mathrm{AP}$ & MPA & $\mathrm{MC}$ & PR & $\mathrm{AM}$ & NMP & IB & $\mathrm{SB}^{*}$ & CLF & LLF & $\mathrm{CP}$ \\
\hline BGMC 788 & $2,43 \mathrm{~A}^{* *}$ & $22669 \mathrm{~F}$ & $7529 \mathrm{D}$ & $22348 \mathrm{E}$ & $32,61 \mathrm{~A}$ & $18,47 \mathrm{~B}$ & $0,05 \mathrm{E}$ & $1,22 \mathrm{C}$ & $19,31 \mathrm{~B}$ & $5,70 \mathrm{~A}$ & $33,17 \mathrm{~B}$ \\
\hline BGMC 1286 & $1,67 \mathrm{~B}$ & $20657 \mathrm{G}$ & $5528 \mathrm{~F}$ & $40423 \mathrm{~B}$ & $29,52 \mathrm{D}$ & $31,42 \mathrm{~A}$ & $0,06 \mathrm{E}$ & $1,50 \mathrm{C}$ & $17,64 \mathrm{C}$ & $5,56 \mathrm{~A}$ & $34,49 \mathrm{~B}$ \\
\hline BGMC 991 & $2,40 \mathrm{~A}$ & $33289 \mathrm{C}$ & $10720 \mathrm{~B}$ & $32095 \mathrm{D}$ & $29,98 \mathrm{C}$ & $37,13 \mathrm{~A}$ & $0,52 \mathrm{~B}$ & $3,05 \mathrm{~A}$ & $21,79 \mathrm{~A}$ & $3,89 \mathrm{~B}$ & $38,45 \mathrm{~A}$ \\
\hline BGMC 1270 & $1,60 \mathrm{~B}$ & $14798 \mathrm{I}$ & $6914 \mathrm{D}$ & $22479 \mathrm{E}$ & $30,54 \mathrm{C}$ & $29,19 \mathrm{~A}$ & $0,11 \mathrm{E}$ & $1,50 \mathrm{C}$ & $13,99 \mathrm{D}$ & $3,78 \mathrm{~B}$ & $23,83 \mathrm{E}$ \\
\hline BGMC 1297 & $2,27 \mathrm{~A}$ & $26870 \mathrm{E}$ & $6089 \mathrm{E}$ & $31966 \mathrm{D}$ & $30,09 \mathrm{C}$ & $11,09 \mathrm{~B}$ & $0,22 \mathrm{D}$ & $1,78 \mathrm{C}$ & $18,31 \mathrm{C}$ & $4,19 \mathrm{~B}$ & $32,36 \mathrm{~B}$ \\
\hline BGMC 996 & $2,47 \mathrm{~A}$ & $42040 \mathrm{~B}$ & $9630 \mathrm{C}$ & $23987 \mathrm{E}$ & $31,97 \mathrm{~B}$ & $11,26 \mathrm{~B}$ & $0,13 \mathrm{E}$ & $1,39 \mathrm{C}$ & $21,00 \mathrm{~A}$ & $3,41 \mathrm{C}$ & $36,99 \mathrm{~A}$ \\
\hline BGMC 1304 & $2,60 \mathrm{~A}$ & 40947 B & $13385 \mathrm{~A}$ & $36629 \mathrm{C}$ & $32,47 \mathrm{~A}$ & $37,50 \mathrm{~A}$ & $0,72 \mathrm{~A}$ & $3,02 \mathrm{~A}$ & 19,53 B & $5,51 \mathrm{~A}$ & $27,93 \mathrm{D}$ \\
\hline BGMC 1265 & $1,53 \mathrm{~B}$ & $18145 \mathrm{H}$ & $7322 \mathrm{D}$ & $24323 \mathrm{E}$ & $29,13 \mathrm{D}$ & $13,30 \mathrm{~B}$ & $0,15 \mathrm{E}$ & $1,61 \mathrm{C}$ & $20,41 \mathrm{~A}$ & $3,08 \mathrm{C}$ & $30,65 \mathrm{C}$ \\
\hline BGMC 1262 & $1,57 \mathrm{~B}$ & $17199 \mathrm{H}$ & $6592 \mathrm{E}$ & $25799 \mathrm{E}$ & $31,89 \mathrm{~B}$ & $13,11 \mathrm{~B}$ & $0,34 \mathrm{C}$ & $2,79 \mathrm{~A}$ & $20,07 \mathrm{~B}$ & $2,49 \mathrm{D}$ & $26,89 \mathrm{E}$ \\
\hline BGMC 923 & $2,17 \mathrm{~A}$ & $30787 \mathrm{D}$ & $13194 \mathrm{~A}$ & $45000 \mathrm{~A}$ & $32,27 \mathrm{~A}$ & $17,42 \mathrm{~B}$ & $0,24 \mathrm{D}$ & $2,17 \mathrm{~B}$ & $20,57 \mathrm{~A}$ & $3,28 \mathrm{C}$ & $35,43 \mathrm{~B}$ \\
\hline BGMC 1298 & $2,27 \mathrm{~A}$ & $24043 \mathrm{~F}$ & $9208 \mathrm{C}$ & $20761 \mathrm{E}$ & $31,39 \mathrm{~B}$ & $17,39 \mathrm{~B}$ & $0,07 \mathrm{E}$ & $1,28 \mathrm{C}$ & $18,42 \mathrm{C}$ & $4,41 \mathrm{~B}$ & $25,53 \mathrm{E}$ \\
\hline BGMC 1130 & $1,73 \mathrm{~B}$ & $19372 \mathrm{G}$ & 7443 D & $26148 \mathrm{E}$ & $31,56 \mathrm{~B}$ & $18,14 \mathrm{~B}$ & $0,14 \mathrm{E}$ & $1,50 \mathrm{C}$ & $18,59 \mathrm{C}$ & $5,51 \mathrm{~A}$ & $28,03 \mathrm{D}$ \\
\hline BGMC 1299 & $2,27 \mathrm{~A}$ & $20535 \mathrm{G}$ & $6916 \mathrm{D}$ & $24000 \mathrm{E}$ & $33,63 \mathrm{~A}$ & $42,41 \mathrm{~A}$ & $0,11 \mathrm{E}$ & $1,39 \mathrm{C}$ & $21,43 \mathrm{~A}$ & $5,51 \mathrm{~A}$ & $28,60 \mathrm{D}$ \\
\hline BGMC 436 & $1,93 \mathrm{~B}$ & $25835 \mathrm{E}$ & $7839 \mathrm{D}$ & $40289 \mathrm{~B}$ & $31,03 \mathrm{~B}$ & $14,92 \mathrm{~B}$ & $0,65 \mathrm{~A}$ & $2,76 \mathrm{~A}$ & $17,69 \mathrm{C}$ & $4,05 \mathrm{~B}$ & $25,91 \mathrm{E}$ \\
\hline BGMC 1107 & $1,07 \mathrm{C}$ & $4031 \mathrm{~J}$ & $3028 \mathrm{G}$ & $8850 \mathrm{~F}$ & $28,51 \mathrm{D}$ & $12,63 \mathrm{~B}$ & $0,05 \mathrm{E}$ & $1,36 \mathrm{C}$ & $20,57 \mathrm{~A}$ & $3,73 \mathrm{~B}$ & $28,16 \mathrm{D}$ \\
\hline BGMC 1305 & $2,37 \mathrm{~A}$ & $46462 \mathrm{~A}$ & $11063 \mathrm{~B}$ & $31333 \mathrm{D}$ & $31,48 \mathrm{~B}$ & $7,84 \mathrm{~B}$ & $0,02 \mathrm{E}$ & $1,28 \mathrm{C}$ & $20,89 \mathrm{~A}$ & $5,81 \mathrm{~A}$ & $33,41 \mathrm{~B}$ \\
\hline Média geral & 2,02 & 25480 & 8275 & 28527 & 31,13 & 20,83 & 0,22 & 1,85 & 19,38 & 4,37 & 30,62 \\
\hline Amplitude $^{\#}$ & 1,53 & 42431 & 10357 & 36150 & 5,12 & 34,57 & 0,70 & 1,83 & 7,8 & 3,32 & 14,62 \\
\hline \multicolumn{12}{|l|}{$\mathrm{FV}(\mathrm{GL})$} \\
\hline $\mathrm{QM}_{\text {Acessos }}(15)$ & $0,53^{+}$ & $367296456^{+}$ & $359657^{+}$ & $248685533^{+}$ & $5,88^{+}$ & $360^{+}$ & $0.14^{+}$ & $0.17^{+}$ & $11.40^{+}$ & $3.53^{+}$ & $57.92^{+}$ \\
\hline $\mathrm{QM}_{\text {Residuo }}(30)$ & 0,05 & 2081900 & 23100151 & 4345318 & 0,46 & 39,02 & 0,003 & 0,01 & 0,44 & 0,09 & 1,78 \\
\hline CV (\%) & 10,92 & 5,66 & 5,58 & 7,31 & 2,18 & 29,99 & 26,34 & 6,68 & 3,43 & 6,72 & 4,36 \\
\hline
\end{tabular}

* = ANOVA realizada com médias transformadas e médias originais apresentadas na tabela;

${ }^{* *}=$ Médias seguidas de letras distintas na coluna pertencem a grupos distintos a 5\% de significância pelo teste de Scott e Knott;

\# = diferença entre a maior e a menor média;

${ }^{+}=$significativo a $5 \%$ de probabilidade de erro pelo teste $\mathrm{F}$.

Fonte: Elaboração dos autores.

\section{Resultados e Discussão}

A análise de variância evidenciou a existência de diferenças significativas entre os acessos para todos os caracteres quantitativos aferidos segundo o teste $\mathrm{F}(\mathrm{P}<0,05)$ (Tabela 2). Esses resultados revelam que no grupo de acessos avaliado, existe variabilidade fenotípica e que a mesma deve ser considerada quando da definição de estratégias de conservação de germoplasma e de uso no melhoramento genético de mandioca. A existência de ampla variabilidade fenotípica era esperada uma vez que foram avaliados acessos de diferentes origens, incluindo acessos melhorados e não melhorados (Tabela 1), como relatado em mandioca por Elias et al. (2001); Nick et al. (2008); Vieira et al. (2008a). Outro importante indicativo da existência de ampla variabilidade quanto aos caracteres quantitativos são as elevadas amplitudes das médias de todos os caracteres aferidos (Tabela 2). Os coeficientes de variação variaram de 2,18\% para o caráter AM a $29,99 \%$ para o caráter NMP, conferindo elevada precisão ao ensaio (Tabela 2). 
Dentre os caracteres quantitativos avaliados, o que apresentou o maior número de classes distintas no teste de comparação de médias (dez classes) foi o caráter MPA, cujas médias variaram de 46462 a $4031 \mathrm{~kg} \mathrm{ha}^{-1}$, para os acessos BGMC 1305 e BGMC 1107, respectivamente (Tabela 2). A MPA é caráter crítico na indicação para o cultivo de acessos de mandioca, uma vez que indica o potencial de produção de manivas-sementes e a possibilidade de utilização da parte área na alimentação animal como fonte protéica (EL-SHARKAWY, 2003; CEBALLOS et al., 2004). O caráter NMP foi o que apresentou o menor número de classes no teste de comparação de médias (duas classes), cujas médias variaram de 4241 a 784 para os acessos BGMC 1299 e BGMC 1305, respectivamente (Tabela 2). A existência de variação quanto ao NMP é importante na possibilidade de utilização de germoplasma no melhoramento de mandioca, uma vez que sugere a existência de níveis distintos de sensibilidade ao percevejo-de-renda Vatiga illudens (Drake, 1922) (Hemiptera: Tingidae), que é uma importante praga da mandioca, e que pode ocasionar redução de até $35 \%$ na PR em acessos susceptíveis (FIALHO et al., 2009). Os caracteres de importância agronômica em mandioca IB e SB que são reflexos da sensibilidade dos acessos à bacteriose causada pela bactéria Xanthomonas axonopodis pv manihotis, que é a principal moléstia que ataca a cultura na região do Cerrado e Centro-Sul do Brasil, também revelaram ampla variação, o que aponta para a existência de fontes de resistência a moléstia no grupo de acessos (Tabela 2).

Os caracteres PR e AM que são os que definem o potencial de produção de fécula de um acesso, apresentaram variações expressivas, com amplitudes de variação de $36150 \mathrm{~kg} \mathrm{ha}^{-1}$ e de $5,12 \%$ para PR e AM, respectivamente, o que é reflexo direto de diferenças no potencial genético dos acessos e no grau de adaptação dos mesmos as condições do Cerrado (Tabela 2). Em especial, se considerarmos que a PR média na região do Cerrado é de 13000 $\mathrm{kg} \mathrm{ha}^{-1}$, e que uma variação de $5,12 \%$ no AM pode representar perda ou ganho de $512 \mathrm{~kg}$ de amido em cada $10000 \mathrm{~kg}$ de raízes de mandioca.

As distâncias estimadas por meio dos caracteres quantitativos variaram de 48 a 2010, sendo a menor distância observada entre os acessos BGMC 986 e BGMC 1130, ambas variedades melhoradas geradas pelo Instituto Agronômico de Campinas (IAC) (Tabela 1 e Figura 1A). Por sua vez, a maior distância foi observada entre os acessos BGMC 1107 e BGMC 1304, uma variedade local do Estado da Paraná e um híbrido gerado pelo programa de melhoramento da Embrapa Mandioca e Fruticultura Tropical (CNPMF) (Tabelas 1, 2 e Figura 1). A análise da Figura 1A revelou a formação de dois agrupamentos sendo o grupo 1 formado pelos acessos BGMC 986, BGMC 1130, BGMC 1299, BGMC 1298, BGMC 1270, BGMC 1297, BGMC 1286, BGMC 1107, BGMC 1265, BGMC 1262 e BGMC 436 e o grupo 2 formado pelos acessos BGMC 991, BGMC 923, BGMC 1304, BGMC 996 e BGMC 1305. O coeficiente de correlação cofenética do dendrograma $(r=0,75)$ revelou um considerável ajuste entre a representação gráfica das distâncias e a matriz de distância de Mahalamobis, o que assegura as inferências realizadas por meio da avaliação visual da Figura 1A.

Dentre os 33 caracteres qualitativos avaliados, 26 (79\%) apresentaram polimorfismo, o que revela a existência de ampla divergência morfológica e a eficiência desses na estimativa da dissimilaridade genética em mandioca, como já havia sido reportado por Elias et al. (2001); Mkumbira et al. (2003) e Vieira et al. (2008a). Apenas sete caracteres qualitativos (21\%) não revelaram variação, uma vez que todos os acessos evidenciaram cor da polpa da raiz branca, poucas constrições nas raízes, pecíolo na posição horizontal, estípulas longas e lancinadas, hábito de crescimento do caule reto e destaque da película da raiz fácil. A dissimilaridade estimada por meio dos caracteres qualitativos variou de 0,19 a 0,73 revelando como acessos mais similares BGMC 1298 e BGMC 1304, dois híbridos gerados pelo programa de melhoramento do CNPMF e, como 
menos similares três pares de acessos BGMC 986 (cultivar do IAC) x BGMC 991 (híbrido CNPMF); BGMC 1107 (variedade local do Estado do Paraná conhecida popularmente como Fécula Branca) x BGMC 986 (cultivar do IAC); e BGMC 991 (híbrido CNPMF) x BGMC 1299 (híbrido CNPMF) (Tabela 1, Figura 1B).

Na Figura 1B baseada nos caracteres qualitativos, verifica-se a formação de três grupos de acessos: i) BGMC 986 e BGMC 1299; ii) BGMC 1286, BGMC 1270, BGMC 1297, BGMC 1107, BGMC 1265, BGMC 1130 e BGMC 1262; e iii) BGMC 991, BGMC 996, BGMC 1304, BGMC 1298, BGMC 923, BGMC 1305 e BGMC 436 (Figura 1B), não sendo observada uma clara tendência de agrupamento dos acessos em função de sua procedência (Tabela 1). O elevado coeficiente de correlação cofenética do dendrograma $(r=0,80)$ revelou elevado ajuste entre a representação gráfica da dissimilaridade e a matriz de dissimilaridade original, conferindo confiabilidade às inferências realizadas por meio da avaliação visual da Figura $1 \mathrm{~B}$.
Os 11 iniciadores utilizados geraram um total de 118 marcadores moleculares RAPD, dos quais 70 (59\%) foram polimórficos, evidenciando a existência de ampla variabilidade genética entre os acessos avaliados (Tabela 3). A elevada eficiência da técnica de marcadores RAPD na discriminação de acessos de mandioca já havia sido relatada por Asante e Offei (2003); Zacarias et al. (2004) e Vieira et al. (2008a), que encontraram porcentagens de polimorfismo de $97,5 \%, 84,6 \%$ e $73 \%$, respectivamente. Dentre os iniciadores empregados no estudo, o que revelou o maior número de bandas polimórficas foi o OPF-08 com 14 bandas, ao passo que o iniciador OPH-13 não apresentou nenhuma banda polimórfica (Tabela 3). A análise do número mínimo de marcadores informativos revelou ser necessária a utilização de pelo menos 65 marcadores polimórficos para se alcançar uma correlação de $r$ $=0,95$ com a utilização de todos os marcadores de forma conjunta, o que justifica a utilização dos 70 marcadores polimórficos obtidos na estimativa da dissimilaridade genética. 
Figura 1. Dendrogramas resultantes da análise de agrupamento de 16 acessos de mandioca de indústria, obtidos pelo método UPGMA, por meio: A) da distância de Mahalanobis (11 caracteres quantitativos) com coeficiente de correlação cofenética $(\mathrm{r}=0,75)$; B) do complemento do índice de coincidência simples (26 caracteres qualitativos) com coeficiente de correlação cofenética $(\mathrm{r}=0,80)$; e C) do complemento do índice de similaridade de Jaccard obtido a partir de 70 marcadores RAPD, com coeficiente de correlação cofenética $(r=0,95)$.

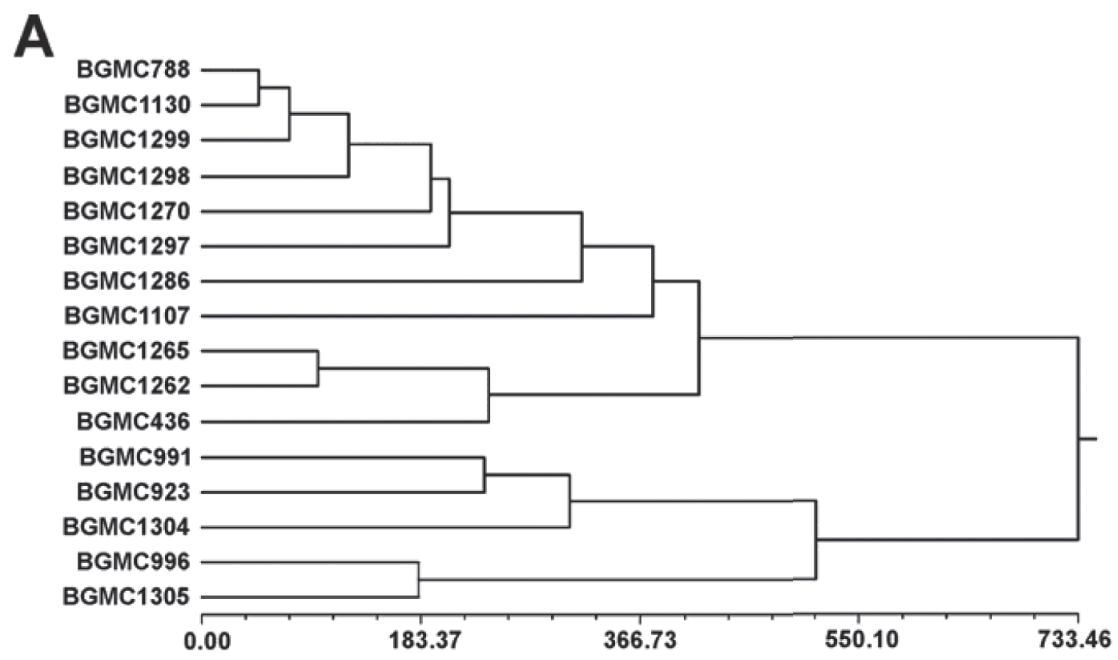

B
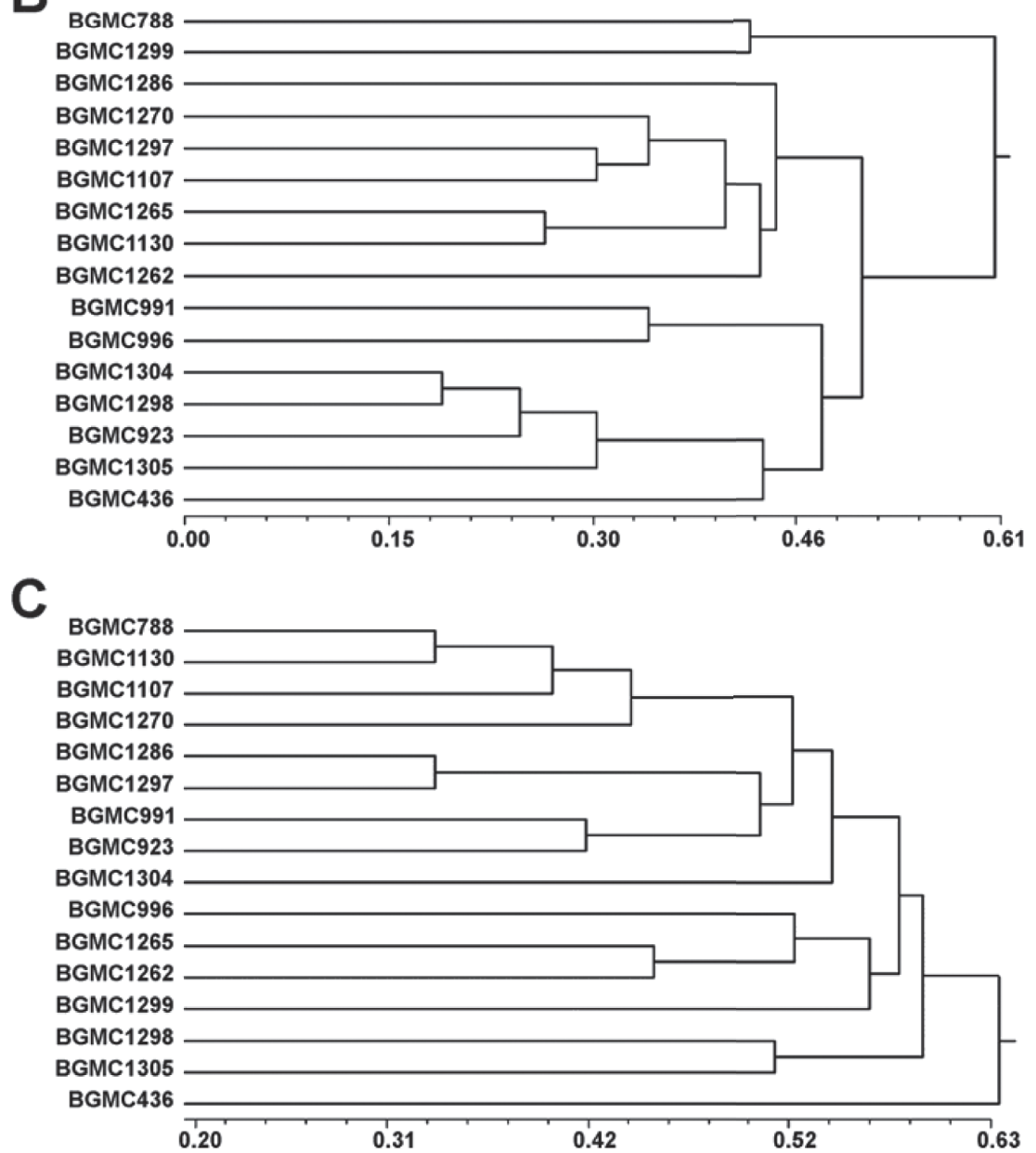

Fonte: Elaboração dos autores. 
Tabela 3. Iniciadores utilizados para obtenção dos marcadores RAPD, respectivo número de bandas polimórficas (BP), número de bandas monomórficas (BM), conteúdo de informação de polimorfismo (CIP) e indíce do marcador (IM).

\begin{tabular}{lccc}
\hline Iniciadores & $\begin{array}{c}\text { Seqüência } \\
5^{\prime} \circledR 3^{\prime}\end{array}$ & BP & BM \\
\hline OPD-02 & GGACCCAACC & 5 & 3 \\
OPE-12 & TTATCGCCCC & 9 & 4 \\
OPF-08 & GGGATATCGG & 14 & 1 \\
OPG-05 & CTGAGACGGA & 5 & 6 \\
OPG-08 & TCACGTCCAC & 5 & 5 \\
OPG-09 & CTGACGTCAC & 8 & 3 \\
OPG-15 & ACTGGGACTC & 13 & 3 \\
OPG-16 & AGCGTCCTCC & 3 & \multicolumn{2}{c}{7} \\
OPH-04 & GGAAGTCGCC & 0 & 5 \\
OPH-13 & GACGCCACAC & 4 & 5 \\
OPH-15 & AATGGCGCAG & 70 & 48 \\
\hline TOTAL & & \multicolumn{2}{c}{} \\
\hline
\end{tabular}

Fonte: Elaboração dos autores.

A dissimilaridade genética estimada por meio de marcadores RAPD variou de 0,33 a 0,72, sendo os pares de acessos mais similares BGMC 1286 (variedade local do Estado de Santa Catarina conhecida como roxinha) x BGMC 1297 (cultivar do CNPMF chamada BRS Mani-branca) e BGMC 1130 (cultivar do IAC chamada IAC 13) x BGMC 986 (cultivar do IAC chamada IAC 14), e que os mais dissimilares foram BGMC 436 e BGMC 996, cultivar do IAC chamada IAC 12 e híbrido do CNPMF, respectivamente (Tabela 1, Figura 1C).

A Figura 1C baseada nos marcadores RAPD, revelou a formação de quatro grupos de acessos: i) BGMC 986, BGMC 1130, BGMC 1107, BGMC 1270, BGMC 1286, BGMC 1297, BGMC 991, BGMC 923 e BGMC 1304; ii) BGMC 996, BGMC 1265, BGMC 1262 e BGMC 1299; iii) BGMC 1298 e BGMC 1305; e iv) BGMC 436 (Figura 1C).
Fato interessante foi a elevada divergência genética apresentada pelo acesso BGMC 436, que é a cultivar de mandioca IAC 12 que apresenta elevado potencial produtivo na região do Cerrado Brasileiro (VIEIRA et al., 2008a; OTSUBO et al., 2009), em relação os demais acessos avaliados (Figura 1C). O coeficiente de correlação cofenética do dendrograma $(r=0,77)$ revelou o considerável ajuste entre a representação gráfica da dissimilaridade genética e a matriz original, o que assegura inferências realizadas por meio da avaliação visual da Figura 1C.

As correlações entre as matrizes de distância/ dissimilaridade estimadas por meios de caracteres quantitativos $\left(\mathrm{MD}_{\mathrm{QT}}\right)$, qualitativos $\left(\mathrm{MD}_{\mathrm{QL}}\right) \mathrm{e}$ moleculares $\left(\mathrm{MD}_{\mathrm{RAPD}}\right)$ revelaram a ausência de associação entre estas estimativas (Tabela 4), como pode também ser observado por meio de inspeção visual das Figuras 1A, 1B e 1C. 
Tabela 4. Correlações entre as matrizes de distância genética, estimadas por meio das análises de caracteres quantitativos $\left(\mathrm{MD}_{\mathrm{QT}}\right)$, qualitativos $\left(\mathrm{MD}_{\mathrm{QL}}\right)$, marcadores RAPD $\left(\mathrm{MD}_{\mathrm{RAPD}}\right)$ e da análise conjunta de caracteres qualitativos, quantitativos e marcadores RAPD ( $\left.\mathrm{MD}_{\mathrm{QT}+\mathrm{QL}+\mathrm{RAPD}}\right)$, entre 16 acessos de mandioca de indústria.

\begin{tabular}{lcccc}
\hline & $\mathrm{MD}_{\mathrm{QT}}$ & $\mathrm{MD}_{\mathrm{QL}}$ & $\mathrm{MD}_{\mathrm{RAPD}}$ & $\mathrm{MD}_{\mathrm{QT}+\mathrm{QL}+\mathrm{RAPD}}$ \\
\hline $\mathrm{MD}_{\mathrm{QT}}$ & 1 & 0,13 & 0,07 & 0,15 \\
$\mathrm{MD}_{\mathrm{QL}}$ & & 1 & 0,01 & $0,52^{*}$ \\
$\mathrm{MD}_{\mathrm{RAPD}}$ & & & 1 & $0,75^{*}$ \\
$\mathrm{MD}_{\mathrm{QT}+\mathrm{QL}+\mathrm{RAPD}}$ & & & 1 \\
\hline
\end{tabular}

${ }^{*}$ Correlação significativa a $1 \%$ de probabilidade de erro, pelo teste de Mantel com 1000 permutações.

Fonte: Elaboração dos autores.

Dentre as matrizes de distância/dissimilaridade estimadas, apenas apresentaram correlações significativas $\mathrm{MD}_{\mathrm{QT}+\mathrm{QL}+\mathrm{RAPD}} \operatorname{com} \mathrm{MD}_{\mathrm{QL}}(\mathrm{r}=0,52)$ e $\mathrm{MD}_{\mathrm{QT}+\mathrm{QL}+\mathrm{RAPD}}$ com $\mathrm{MD}_{\mathrm{RAPD}}(\mathrm{r}=0,75)$, enquanto que as demais matrizes de distância/dissimilaridade apresentaram correlações reduzidas ou nulas (Tabela 4). Esta ausência de associação entre as estimativas não deve ser considerada como uma limitação dessas ferramentas de acesso à variabilidade genética, mas sim como um indicativo da complementaridade entre as mesmas (LEFEBVRE et al., 2001; RANA; SINGH; BHAT, 2005; LI et al., 2008; SILVA et al., 2009), podendo ser explicada pelas diferentes propriedades dos marcadores moleculares e dos caracteres qualitativos e quantitativos. Normalmente, a maior parte da variação detectada por marcadores moleculares é do tipo não adaptativa e, portanto, não sujeita à seleção natural e/ou artificial, ao contrário dos caracteres fenotípicos que na sua maioria são sujeitos a seleção natural e/ ou artificial, em especial os caracteres quantitativos e qualitativos de interesse agronômico e/ou adaptativo (VIEIRA et al., 2007). Uma variação em nível de DNA nem sempre é reflexo de variações fenotípicas e a ausência de variação fenotípica, nem sempre e sinal de ausência de variação em nível de DNA, uma vez que diferentes genes podem levar a expressão de um mesmo fenótipo (RANA; SINGH; BHAT, 2005). Desta forma, é possível estabelecer um cenário em que as associações entre as distâncias/dissimilaridades genéticas estimadas por meio de caracteres qualitativos, quantitativos e moleculares serão tão mais próximas quanto maior for a associação entre os locos que controlam os caracteres quantitativos e qualitativos estudados e as bandas avaliadas e quanto maior for o número de caracteres quantitativos e qualitativos avaliados, o que aumentará a cobertura do genoma dos acessos (LEFEBVRE et al., 2001; MÁRIC et al., 2004; ROY et al., 2004; VIEIRA et al., 2007).

Em estudo que avaliou a variabilidade genética entre acessos de mandioca açucarados e não açucarados, Vieira et al. (2008a) relataram a ocorrência de associação elevada entre as distâncias estimadas por meio de marcadores RAPD e caracteres qualitativos $(r=0,83)$ e moderada entre as distâncias estimadas por meio de caracteres qualitativos e quantitativos $(r=0,50)$ e entre marcadores RAPD e caracteres quantitativos $(\mathrm{r}=$ $0,58)$. Entretanto, cabe ressaltar que provavelmente esta elevada associação seja decorrência do fato dos autores terem avaliado acessos pertencentes a pool gênicos muito dissimilares, mandiocas açucaradas e não açucaradas o que favoreceu a ocorrência de elevada associação. Por outro lado, em estudos com diversas espécies vem sendo relatada baixas correlações entre estimativas de distâncias genéticas estimadas por meio de marcadores moleculares e caracteres fenotípicos (RANA; SINGH; BHAT, 2005; GELETA; LABUSCHAGNE; VILJOEN, 2006; LI et al., 2008). Tendo em vista a complementaridade das técnicas, uma alternativa é o emprego de marcadores moleculares, caracteres qualitativos, caracteres quantitativos de 
forma conjunta e complementar, a fim de orientar estratégias de conservação e de uso de germoplasma.

A análise conjunta com marcadores RAPD, caracteres morfológicos e caracteres quantitativos revelou dissimilaridades genéticas que variaram de 0,26 a 0,50, sendo os acessos mais similares BGMC 1286 (variedade local do Estado de Santa Catarina) e BGMC 1297 (cultivar melhorada pelo CNPMF para a região nordeste do Brasil chamada BRS Manibranca) e os mais dissimilares BGMC 436 (cultivar do IAC chamada IAC 12) e BGMC 996 (híbrido do CNPMF) (Figura 2). A Figura 2 revelou a formação de cinco grupos de acessos: i) BGMC 986 e BGMC
1299; ii) BGMC 1286, BGMC 1297, BGMC 1270, BGMC 1265, BGMC 1107 e BGMC 1130; iii) BGMC 991, BGMC 996, BGMC 923, BGMC 1304, BGMC 1298 e BGMC 1305; iv) BGMC 1262; e v) BGMC 436 (Figura 2). Fato interessante foi a elevada divergência genética apresentada pelos acessos BGMC 436 e BGMC 1262 em relação os demais acessos avaliados (Figura 2). O coeficiente de correlação cofenética do dendrograma $(r=0,81)$ revelou o considerável ajuste entre a representação gráfica da dissimilaridade genética e a matriz original, o que assegura inferências realizadas por meio da avaliação visual da Figura 2.

Figura 2. Dendrograma resultante da análise de agrupamento de 16 acessos de mandioca de indústria, obtido pelo método UPGMA, utilizando o complemento do índice de similaridade de Gower (1971), obtido a partir da análise conjunta de caracteres quantitativos, qualitativos e marcadores RAPD, como medida de distância genética. $\mathrm{O}$ valor do coeficiente de correlação cofenética (r) é de 0,81 .

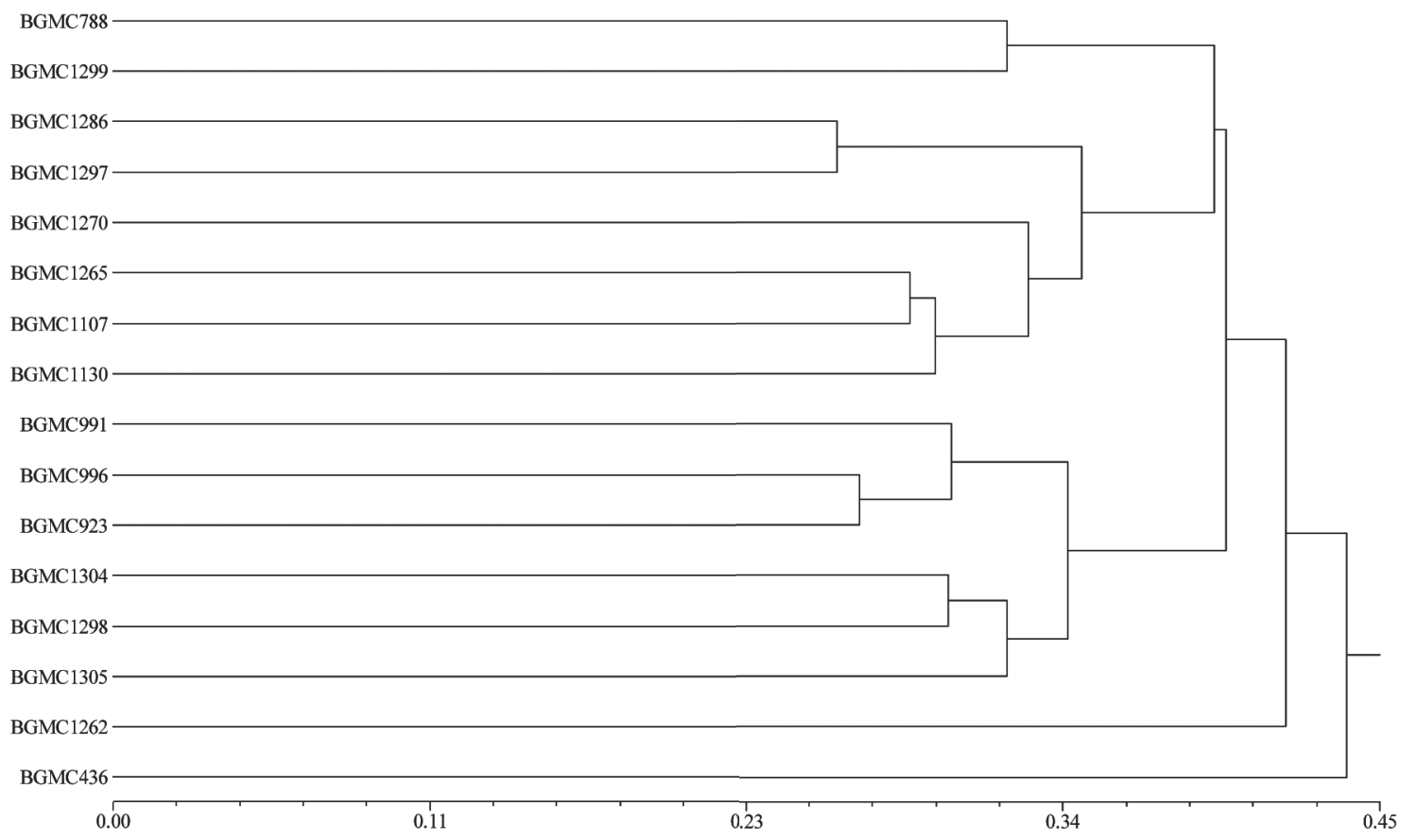

Fonte: Elaboração dos autores. 
A matriz de dissimilaridade genética estimada por meio da análise conjunta de caracteres quantitativos, caracteres qualitativos e marcadores moleculares $\left(\mathrm{MD}_{\mathrm{QT}+\mathrm{QL}+\mathrm{RAPD}}\right)$, revelou correlação significativa moderada com $\mathrm{MD}_{\mathrm{QL}}(\mathrm{r}=0,52)$ e elevada com $\operatorname{MD}_{\text {RAPD }}(r=0,75)$, enquanto que apresentou correlação reduzida com $\mathrm{MD}_{\mathrm{QT}}$ ( $\mathrm{R}$ $=0,15)$ (Tabela 4). Tal diferença na associação entre as matrizes provavelmente tenha por base o fato de ter sido utilizado na análise conjunta um número maior de marcadores RAPD (70) do que de caracteres quantitativos (13) e caracteres qualitativos (26). Esses resultados apontam no sentido de que a análise conjunta de dados é mais eficiente quando mais balanceado for o número de caracteres/marcadores disponíveis entre as diferentes ferramentas de acesso a variabilidade genética disponíveis. Nesse sentido, Vieira et al. (2007) estabeleceram a hipótese de que para que se obtenha um conhecimento mais completo do grau de divergência entre acessos é necessário considerar separadamente as análises com dados moleculares e fenotípicos, para que então, seja possível chegar a uma conclusão final a respeito da divergência genética apresentada entre os acessos analisados. Entretanto, este estudo evidenciou que a possibilidade de utilização de um índice de dissimilaridade de forma conjunta paralelamente a análise individuais das dissimilaridades contribui para a interpretação dos resultados e é uma ferramenta auxiliar de grande importância em estudos de conservação e uso de germoplasma.

Do ponto de vista prático, o estudo com base nos diferentes grupos de caracteres quantitativos, qualitativos e moleculares revelou a existência de elevada variabilidade genética entre os acessos analisados (Tabelas 2 e 3, Figuras 1A, 1B, 1C e 2). $\mathrm{O}$ fato de ter sido detecta ampla variabilidade genética mesmo entre acessos melhorados, é consequência direta da utilização da ampla base genética de mandioca disponível no Brasil (VIEIRA et al., 2008b) de forma constante no melhoramento genético da cultura. Estudos tem demonstrado, que o fato da mandioca apresentar sistema reprodutivo que favorece a fecundação cruzada, que permite uma constante recombinação gênica, aliada a propagação vegetativa, tem reflexo direto na capacidade de adaptação da espécie às mudanças ambientais (CEBALLOS et al., 2004; EL-SHARKAWY, 2004; DUPUTIÉ et al., 2009).

Em relação à conservação e uso do germoplasma, o estudo revelou que no grupo de acessos avaliados existe variabilidade genética suficiente para o estabelecimento de um programa de melhoramento genético voltado à geração variedades de mandioca de indústria adaptadas as condições do Cerrado do Brasil Central.

\section{Conclusões}

No grupo de acessos de indústria avaliados existe ampla variabilidade quanto aos caracteres quantitativos, qualitativos e moleculares aferidos.

A melhor estratégia para orientar ações de conservação e uso de germoplasma de mandioca é por meio de estudos de divergência genética com o emprego de marcadores moleculares, caracteres qualitativos e caracteres quantitativos de forma conjunta e complementar.

\section{Agradecimentos}

Os autores agradecem a Embrapa, a Fundação Banco do Brasil e ao CNPq pelo apoio financeiro.

\section{Referências}

ASANTE, I. K.; OFFEI, S. K. RAPD-based genetic diversity study of fifty cassava (Manihot esculenta Crantz) genotypes. Euphytica, Wageningen, v. 131, n. 1, p. 113-119, 2003.

CARVALHO, L. J. C. B.; SCHAAL, B. A. Assessing genetic diversity in the cassava (Manihot esculenta Crantz) germoplasm collection in Brazil using PCRbased Markers. Euphytica, Wageningen, v. 120, n. 1, p. 133-142, 2001. 
CEBAlloS, H.; IGLESIAS, C. A.; PÉREZ, J. C.; DIXON, A. G. O. Cassava breeding: opportunities and challenges. Plant Molecular Biology, Zurich, v. 56, n. 4, p. 503-516, 2004.

COSTA, A. M.; FALEIRO, F. G.; KARIA, C. T.; SHIRATSUCHI, L. S.; ANDRADE, R. P.; LOPES, G. K. B. Variabilidade genética e ecológica de Stylosanthes macrocephala determinadas por RAPD e SIG. Pesquisa Agropecuária Brasileira, Brasília, v. 40, n. 9, p. 899-909, 2005.

CRUZ, C. D. Programa genes: aplicativo computacional em genética e estatística. Viçosa: UFV, 2001. 648 p.

DUPUTIÉ, A.; MASSOL, F.; DAVID, P.; HAXAIRE, C.; McKEY, D. Traditional Ameridian cultivators combine directional and ideotypic selection for sustaineble management of cassava genetic diversity. Journal of Evolutionary Biology, Basel, v. 22, n. 6, p. 1317-1325, 2009.

ELIAS, M.; McKEY, D.; PANAUD, O.; ANSTETT, M. C.; ROBERT, T. Traditional management of cassava morphological and genetic diversity by the Makushi Amerindians (Guyana, South America): Perspectives for on-far conservation of crop genetic resources. Euphytica, Wageningen, v. 120, n. 1, p. 143-157, 2001.

EL-SHARKAWY, M. A. Cassava biology and physiology. Plant Molecular Biology, Zurich, v. 56, n. 4, p. 481-501, 2004.

FALEIRO, F. G.; FALEIRO, A. S. G.; CORDEIRO, M. C. R.; KARIA, C. T. Metodologia para operacionalizar a extração de DNA de espécies nativas do cerrado. Planaltina: CPAC, 2003. 6 p.

FERREIRA, C. F.; ALVES, E.; PESTANA, K. N.; JUNGHANS, D. T.; KOBAYASHI, A. K.; SANTOS, V. J.; SILVA, R. P.; SILVA, P. H.; SOARES, E.; KUKUDA, W. Molecular characterization of cassava (Manihot esculenta Crantz) with yellow-orange roots for betacarotene improvement. Crop Breeding and Applied Genetics, Viçosa, v. 8, n. 1, p. 23-29, 2008.

FIALHO, J. F.; VIEIRA, E. A.; MORAES, S. V. P.; SILVA, M. S.; JUNQUEIRA, N. T. V. Danos causados por percevejo-de-renda na produtividade da parte aérea e raízes de mandioca. Scientia Agraria, Curitiba, v. 10, n. 2, p. 151-155, 2009.

FUKUDA, W. M. G.; GUEVARA, C. L. Descritores morfológicos e agronômicos para a caracterização de mandioca (Manihot esculenta Crantz). Cruz das Almas: CNPMF, 1998. 38 p.

GELETA, N.; LABUSCHAGNE, M. T.; VILJOEN, C. D. Genetic diversity analysis in sorghum germplasm as estimated by AFLP, SSR and morpho-agronomical markers. Biodiversity and Conservation, Dordrecht, v. 15, n. 10, p. 3251-3265, 2006.

GOWER, J. C. A general coefficient of similarity and some of its properties. Biometrics, Washington, v. 27, n. 4, p. 857-874, 1971.

JACCARD, P. Nouvelles recherches sur la distribution florale. Bulletin Société Vaudoise des Sciences Naturelles, Lausane, v. 44, n. 1, p. 223-270, 1908.

LEFEBVRE, V.; GOFFINET, B.; CHAUVET, J. C.; CAROMEL, B.; SIGNORET, P.; BRAND, R.; PALLOIX, A. Evaluation of genetic distances between pepper inbred lines for cultivar protection purposes: comparison of AFLP, RAPD, and phenotypic data. Theoretical and Applied Genetics, New York, v. 102, n. 5, p. 741-750, 2001.

LI, F.; GAN, S.; WENG, Q.; ZHAO, X.; HUANG, S.; LI, M.; CHEN, S.; WANG, Q.; SHI, F. RAPD and morphological diversity among four populations of the tropical tree species Paramichelia baillonii (Pierre) $\mathrm{Hu}$ in China. Forest Ecology and Management, Amsterdam, v. 255, n. 5-6, p. 1793-1801, 2008.

MANTEL, N. The detection of disease clustering and a generalized regression approach. Cancer Research, Chestnut, v. 27, n. 2, p. 209-220, 1967.

MÁRIC, S.; BOLARÍC, S.; MARTINCIC, J.; PEJÍC, I.; KOZUMPLINK, V. Genetic diversity of hexaploid wheat cultivars estimated by RAPD markers, morphological traits and coefficients of parentage. Plant Breeding, Berlin, v. 123, n. 4, p. 366-369, 2004.

MKUMBIRA, J.; CHIWONA-KARLTUN, L.; LAGERCRANTZ, U.; MAHUNGU, N. M.; SAKA, J.; MHONE, A.; BOKANGA, M.; BRIMER, L.; GUllBERG, U.; ROSLING, H. Classification of cassava into "bitter" and "cool" in Malawi: from farmer's perception to characterization by molecular markers. Euphytica, Wageningen, v. 132, n. 1, p. 7-22, 2003.

MOHAMMADI, S. A.; PRASANNA, B. M. Analyses of genetic diversity in crop plants - salient statistics tools and considerations. Crop Science, Madisson, v. 43, n. 4, p. $1235-1248,2003$.

NICK, C.; CARVALHO, M.; ASSIS, L. H. B.; CARVALHO, S. P. Genetic dissimilarity in cassava clones determined by multivariate techniques. Crop Breeding and Applied Genetics, Viçosa, v. 8, n. 2, p. 104$110,2008$.

OLSEN, K. M. SNPs, SSRs and inferences on cassava's origin. Plant Molecular Biology, Zurich, v. 56, n. 4, p. 517-526, 2004. 
OTSUBO, A. A.; BRITO, O. R.; MERCANTE, F. M.; OTSUBO, V. H. N.; GONÇALVES, M. A.; TELLES, T. S. Desempenho de cultivares elites de mandioca industrial em área de cerrado do Mato Grosso do Sul. Semina: Ciências Agrárias, Londrina, v. 30, p. 11551162, 2009. Suplemento 1.

PILLAR, V. P. Multivariate exploratory analysis and randomization testing using Multiv. Coenoses, Gorizias, v. 12, n. 1-2, p. 145-148, 1997.

RAMOS, J. G. A.; TAKATSU, A. Avaliação $d a$ resistência de cultivares de mandioca à bacteriose em Goiás. Goiânia: EMGOPA, 1987.26 p.

RANA, M. K.; SINGH, V. P.; BHAT, K. V. Assessment of genetic diversity in upland cotton (Gossypium hirsutum L.) breeding lines by using amplified fragment length polymorphism (AFLP) markers and morphological characteristics. Genetic Resources and Crop Evolution, Dordrecht, v. 52, n. 8, p. 989-997, 2005.

ROHLF, F. J. NTSYS-pc: numerical taxonomy and multivariate analysis system, version 2.1. New York: Exeter Software, 2000. 98 p.

ROY, J. K.; LAKSHMIKUMARAN, M. S.; BALYAN, H. S.; GUPTA, P. K. AFLP-based genetic diversity and its comparison with diversity based on SSR, SAMPL, and phenotypic traits in bread wheat. Biochemical Genetics, New York, v. 42, n. 1-2, p. 43-59, 2004.

SCOTT, A. J.; KNOTT, M. A cluster analysis method for grouping means in the analysis of variance. Biometrics, Washington, v. 30, n. 3, p. 507-512, 1974.

SILVA, G. O.; PEREIRA, A. S.; SOUZA, V. Q.; CASTRO, C. M.; CARFALHO, F. I. F. C.; VIEIRA, E. A. Distâncias genéticas entre genótipos de batata a partir de dados morfológicos, moleculares e genealógicos. Semina: Ciêncisa Agrárias, Londrina, v. 30, p. 983-992, 2009. Suplemento 1.
SOKAL, R. R.; ROHLF, F. J. The comparison of dendrograms by objective methods. Taxon, Berlin, v. 11, n. 1, p. 30-40, 1962.

SOUZA, L. S.; FIALHO, J. F. Sistema de produção de mandioca para a região do cerrado. Cruz da Almas: CNPMF, 2003. $61 \mathrm{p}$.

VIEIRA, E. A., FIALHO, J. F.; FALEIRO, F. G.; BELLON, G.; FONSECA, K. G.; CARVALHO, L. J. C. B.; SILVA, M. S.; MORAES, S. V. P.; FILHO, M. O. S. S., SILVA, K. N. Divergência genética entre acessos açucarados e não açucarados de mandioca. Pesquisa Agropecuária Brasileira, Brasília, v. 43, n. 12, p. 17071715, 2008a.

VIEIRA, E. A.; CARVALHO, F. I. F.; BERTAN, I.; KOPP, M. M.; ZIMMER, P. D.; BENIN, G.; SILVA, J. A. G.; HARTWIG, I.; MALONE, G.; OLIVEIRA, A. C. Association between genetic distances in wheat (Triticum aestivum) as estimated by AFLP and morphological markers. Genetics and Molecular Biology, Ribeirão Preto, v. 30, n. 2, p. 392-399, 2007.

VIEIRA, E. A.; FIALHO, J. F.; SILVA, M. S.; FUKUDA, W. M. G.; FALEIRO, F. G. Variabilidade genética do banco de germoplasma de mandioca da Embrapa Cerrados acessada por meio de descritores morfológicos. Cientifica, Jaboticabal, v. 36, n. 1, p. 56-67, 2008 b.

VILPOUX, O. F. Competitividade da mandioca no Brasil, como matéria prima para amido. Informações Econômicas, São Paulo, v. 38, n. 1, p. 27-38, 2008.

ZACARIAS, A. M.; BOTHA, A. M.; LABUSCHAGNE, M. T.; BENESI, I. R. M. Characterization and genetic distance analysis of cassava (Manihot esculenta Crantz) germplasm form Mozambique using RAPD fingerprinting. Euphytica, Wageningen, v. 138, n. 1, p. 49-53, 2004. 
